\title{
COINTEGRATION AND UNIT ROOTS
}

Juan J. Dolado

Bank of Spain

Tim Jenkinson

Keble College, University of Oxford and CEPR

Simon Sosvilla-Rivero

University of Birmingham

Abstract. This paper provides an updated survey of a burgeoning literature on testing, estimation and model specification in the presence of integrated variables. Integrated variables are a specific class of non-stationary variables which seem to characterise faithfully the properties of many macroeconomic time series. The analysis of cointegration develops out of the existence of unit roots and offers a generic route to test the validity of the equilibrium predictions of economic theories. Special emphasis is put on the empirical researcher's point of view.

Keywords. Unit root, cointegration, trends, error correction mechanisms.

\section{Introduction}

Economic theory generally deals with equilibrium relationships. Most empirica econometric studies are an attempt to evaluate such relationships by summarising economic time series using statistical analysis. To apply standard inference procedures in a dynamic time series model we need the various variables to be stationary, since the majority of econometric theory is built upon variables to be stationary, since the majority of econometric theory is built upon
the assumption of stationarity. Until recently, this assumption was rarely the assumption of stationarity. Until recently, this assumption was rarely questioned, and econometric analysis proceeded as if all the economic time series be appropriately removed. However, stationary series should at least have constant unconditional mean and variance over time, a condition which appears rarely to be satisfied in economics. The importance of the stationarity rarely to be satisfed in economics. The importance of the stapionarity assumption had been recognised for many years, but the important papers by Granger and Newbold (1974), Nelson and Kang (1981) and Nelson and Plosse (1982) alerted many to the econometric implications of non-stationarity. Integrated variables are a specific class of non-stationary variables with important economic and statistical properties. These are derived from the presence of unit roots which give rise to stochastic trends, as opposed to pur deterministic trends, with innovations to an integrated process being permanent 
instead of transient. For example, the presence of a large permanent component in aggregate output conflicts with traditional formulations of both Keynesian and Classical macroeconomic theories in terms of countercyclical policies, implying, in addition, that the welfare costs and benefits of policy actions are far different than when output

The presence of, at least, a unit root is implied by many economic models by the rational use of available information by economic agents. Standard applications include futures contracts, stock prices, yield curves, real interest rates, exchange rates, money velocity, hysteresis theories of unemployment, and, perhaps the most popular, the implications of the permanent income hypothesis for real consumption.

Statisticians have been aware for many years of the existence of integrated series and, in fact, Box and Jenkins (1970) argue that a non-stationary series can be transformed into a stationary one by successive differencing of the series. Therefore, from their point of view, the differencing operation seemed to be a pre-requisite for econometric modelling both from an univariate and a multivariate perspective. However Sargan (1964), Hendry and Mizon (1978) and Davidson et al. (1978), among others, have criticised on a number of grounds the specification of dynamic models in terms of differenced variables only, especially because it is then impossible to infer the long-run steady state solution from the estimated model.

Granger (1983) and Granger and Weiss (1983), resting upon the previous ideas, point out that a vector of variables, all of which achieve stationarity after differencing, may have linear combinations which are stationary without differencing. Engle and Granger (1987) formalise the idea of variables sharing an equilibrium relationship in terms of cointegration between time series, providing us with tests and an estimation procedure to evaluate the existence of equilibrium relationships, as implied by economic theory, within a dynamic specification framework. Standard examples include the relationship between real wages and disposable income, long and short-term interest rates, money velocity and interest rates, production and sales, etc.

In view of this epidemic of martingales in economics, a voluminous literature on testing, estimation, prediction, control and model specification in the presence of integrated variables has developed in the last few years. The purpose of this survey is to provide a useful guide through this increasingly technical literature, paying special attention to the point of view of the applied researcher with a good grounding in econometrics, who being a non-specialist in researcher with a good grounding in econometrics, who being a non-specialist in
this particular subject wants to get a unified coverage of the main techniques available in this field.

The paper is organised as follows. The concepts of cointegration and unit roots are introduced in Section 2. In Section 3 we survey several alternative tests for the existence of unit roots, including cases where seasonality is present. Section 4 deals with alternative definitions of integration. Section 5 examines the application of some of the previous tests to determine the existence of cointegrating relationships. Section 6 contains a review of some new tes procedures for cointegration. Finally, brief conclusions follow in Section 7 .

\section{Unit roots and cointegration}

Wold's (1938) decomposition theorem states that a stationary time series proces with no deterministic component has an infinite moving average (MA) representation. This, in turn, can be represented approximately by a finite autoregressive moving average (ARMA) process (see, e.g. Hannan, 1970)

However, as was mentioned in the Introduction, some time series need to be appropriately differenced in order to achieve stationarity. From this comes the definition of integration (as adopted by Engle and Granger, 1987): A variable $y_{t}$ is said to be integrated of order $d$ [or $\left.y_{t} \sim I(d)\right]$ if it has stationary, invertible, non-deterministic ARMA representation after differencing $d$ times. Thus, a time series integrated of order zero is stationary in levels, while for a time series integrated of order one, the first difference is stationary. A white noise series and a stable first-order autoregressive [AR(1)] process are examples of $I(0)$ series, while a random walk process is an example of an $I(1)$ series.

Granger (1986) and Engle and Granger (1987) discuss the main differences between processes that are $I(0)$ and $I(1)$. They point out that an $I(0)$ series: (i) has finite variance which does not depend on time, (ii) has only a limited memory of its past behaviour (i.e. the effects of a particular random innovation are only transitory), (iii) tends to fluctuate around the mean (which may include a deterministic trend), and (iv) has autocorrelations that decline rapidly as the lag increases. For the case of an $I(1)$ series, the main features are: (i) the variance depends upon time and goes to infinity as time goes to infinity, (ii) the process has an infinitely long memory (i.e. an innovation will permanently affect the process), (iii) it wanders widely, and (iv) the autocorrelations tend to one in magnitude for all time separations.

Consider now two time series $y_{t}$ and $x_{t}$ which are both $I(d)$ (i.e. they have compatible long-run properties). In general, any linear combination of $y_{t}$ and $x_{t}$ will be also $I(d)$. If, however, there exists a vector $(1,-\beta)^{\prime}$, such that the combination

$$
z_{t}=y_{t}-\alpha-\beta x_{t}
$$

is $I(d-b), b>0$, then Engle and Granger (1987) define $y_{t}$ and $x_{t}$ as cointegrated of order $(d, b)$ [or $\left(y_{t}, x_{t}\right)^{\prime} \sim C I(d, b)$ ], with $(1,-\beta)^{\prime}$ called the cointegrating vector. Note that a constant term has been included in (1) in order to allow for the possibility that $z_{t}$ may have a non-zero mean.

The concept of cointegration tries to mimic the existence of a long-run equilibrium to which an economic system converges over time. If, e.g., economic theory suggests the following long-run relationship between $y_{t}$ and $x_{t}$

$$
y_{t}=\alpha+\beta x_{t}
$$


then $z_{t}$ can be interpreted as the equilibrium error (i.e., the distance that the system is away from the equilibrium at any point in time).

Engle and Granger also show that if $y_{t}$ and $x_{t}$ are cointegrated $C I(1,1)$, then there must exist an error correction model (ECM) representation of the following form ${ }^{2}$

$$
\Delta y_{t}=\theta_{0}+\theta_{1} z_{t-1}+\Sigma \theta_{2 i} \Delta x_{t-i}+\Sigma \theta_{3 i} \Delta y_{t-i}+\varepsilon_{t}
$$

where $\Delta$ denotes the first-order time difference (i.e. $\Delta y_{t}=y_{t}-y_{t-1}$ ) and where $\left\{\varepsilon_{t}\right\}$ is a sequence of independent and identically distributed random variables with mean zero and variance $\sigma_{\varepsilon}^{2}$ (i.e. $\varepsilon_{t} \sim i i d\left(0, \sigma_{\varepsilon}^{2}\right)$ ). Furthermore, they prove the converse result that an ECM generates cointegrated series.

Note that the term $z_{t-1}$ in equation (3) represents the extent of the disequilibrium between levels of $y$ and $x$ in the previous period. The ECM states that changes in $y_{t}$ depend not only on changes in $x_{t}$, but also on the extent of disequilibrium between the levels of $y$ and $x$. The appeal of the ECM formulation is that it combines flexibility in dynamic specification with desirable long-run properties: it could be seen as capturing the dynamics of the system whilst incorporating the equilibrium suggested by economic theory (see Hendry and Richard, 1983).

Based upon the concept of cointegration (and on its closely related concept of ECM representation), Engle and Granger suggest a 2-step estimation procedure for dynamic modelling which has become very popular in applied research. Let us assume that $y_{t}$ and $x_{t}$ are both $I(1)$, then the procedure goes as follows:

(i) First, in order to test whether the series are cointegrated, the "cointegrating regression'

$$
y_{t}=\alpha+\beta x_{t}+z_{t}
$$

is estimated by ordinary least squares (OLS) and it is tested whether the 'cointegrating residuals' $\hat{z}_{t}=y_{t}-\hat{\alpha}-\hat{\beta} x_{t}$ are $I(0)$. Stock (1987) has shown that if two $I(1)$ series are cointegrated, then the OLS estimates from equation (4) provide 'super-consistent' estimates of the cointegrating vector, in the sense that they converge to the true parameter at a rate proportional to the inverse sample size, $T^{-1}$, rather than at $T^{-1 / 2}$ as in the ordinary stationary case. ${ }^{4}$ The intuition behind this remarkable result can be seen by analysing the behaviour of the OLS estimator of $\beta$ in (4) (where the constant is eliminated for simplicity), when $z_{t} \sim$ iid $\left(0, \sigma_{z}^{2}\right)$ and $x_{t}$ follows a random walk:

$$
\Delta x_{t}=\varepsilon_{t} ;\left(x_{0}=0, \varepsilon_{t} \sim \operatorname{iid}\left(0, \sigma_{\varepsilon}^{2}\right)\right)
$$

Integrating (5) backwards we get

$$
x_{t}=\sum_{i=1}^{t} \varepsilon_{i}=S_{t}
$$

and therefore $\operatorname{var}\left(x_{t}\right)=t \sigma_{\varepsilon}^{2}$, exploding as $T \uparrow \infty .^{5}$ Nevertheless $T^{-2} \Sigma x_{t}^{2}$ converges to a random variable. Similarly the cross-moment $T^{-1 / 2} \Sigma x_{t} z_{t}$ will explode, in contrast to the stationary case, where it is asymptotically normally distributed. In the $I(1)$ case $T^{-1} \Sigma x_{t} z_{t}$ converges also to a random variable. Both random variables are functionals of Brownian Motions or Wiener processes, which will be denoted henceforth, in general, as $f(W)$ (see Phillips (1987), Phillips and Perron (1988) and Park and Phillips (1988) for a general discussion on convergence of the above mentioned distributional limits). From the expression for the OLS estimator of $\beta$ we obtain

$$
\hat{\beta}-\beta=\Sigma x_{t} z_{t} / \Sigma x_{t}^{2}
$$

it follows from the previous discussion that $T(\hat{\beta}-\beta)$ is asymptotically the ratio of two non-degenerate random variables, and it is in general not normal. Thus, standard inference cannot be applied to $\hat{\beta}$, even if it is 'super-consistent', question to which we will come back in Section 6.

(ii) Finally, the residuals $\hat{z}_{t}$ are entered into the ECM. Now, all the variables in equation (3) are $I(0)$ and conventional modelling strategies can be applied.

\section{Testing the order of integration of the relevant variables}

Once the relevant set of variables suggested by economic theory has been identified, the first stage in testing for cointegration between those variables is to determine the order of integration of the individual time series.

Several statistical tests for unit roots have been developed to test for stationarity in time series. Since many macroeconomic series have been found to be integrated of order one (see, e.g. Nelson and Plosser, 1982), we will only consider tests for a single unit root.

The previous tests can also be applied, with a slight change in their interpretation, for sequential testing of unit roots, i.e. when one wants to compare a null hypothesis of $k$ unit roots with an alternative of $k-1$ unit roots. In the sequential procedure, the investigator should start with the largest $k$ under consideration and work down; that is, decrease $d$ by one each time the null hypothesis is rejected. ${ }^{6}$

3.1. Tests of unit roots

(i) Dickey and Fuller $(1979,1981)$ present a class of test statistics, known as Dickey-Fuller (DF) statistics, generally used to test that a pure AR(1) process (with or without drift) has a unit root.

Let the time series $y_{t}$ satisfy the following data generating process (DGP)

$$
y_{t}=\beta_{0}+\beta_{1} t+\rho y_{t-1}+\varepsilon_{t}
$$

where $\varepsilon_{t} \sim \operatorname{iid}\left(0, \sigma_{\varepsilon}^{2}\right), t$ is a time trend and the initial condition, $y_{0}$, is assumed to be a known constant (zero, without loss of generality). Equation (6) can also be written as

$$
y_{t}=\beta_{0} \sum_{j=1}^{t} \rho^{t-j}+\beta_{1} \sum_{j=1}^{t} j \rho^{t-j}+\sum_{j=1}^{t} \varepsilon_{j} \rho^{t-j}
$$


while in the case that $\rho=1$

$$
y_{t}=\beta_{0} t+\beta_{1} t(t+1) / 2+S_{t}
$$

where $S_{t}=\sum_{j=1}^{t} \varepsilon_{j}$

Dickey and Fuller (1979) consider the problem of testing the null hypothesis $H_{0}: \rho=1$ versus $H_{1}: \rho<1$, i.e. non-stationarity vs. stationarity around a deterministic trend, suggesting OLS estimation of a reparameterised version of (6), i.e.

$$
\Delta y_{t}=\beta_{0}+\beta_{1} t+\gamma y_{t-1}+\varepsilon_{t}
$$

where $H_{0}: \rho=1$ is equivalent to $H_{0}: \gamma=0$ (since $\gamma=\rho-1$ ). The test is implemented though the usual $t$-statistic of $\hat{\gamma}$, denoted here as $\tau_{\tau}$. In addition, Dickey and Fuller (1981) suggest two $F$-statistics for the joint null hypothesis $\beta_{0}=\beta_{1}=\gamma=0$ and $\beta_{1}=\gamma=0$, denoted as $\Phi_{2}$ and $\Phi_{3}$ respectively. Note that under the null hypothesis $\tau_{\tau}, \Phi_{2}$ and $\Phi_{3}$ will not have the standard $t$ and $F$ distributions, instead they are functions of Brownian motion; we must use the asymptotic distributions tabulated in Fuller (1976, p. 373) and in Dickey and Fuller $\left(1981\right.$, p. 1,063) respectively. If $\beta_{1}=0\left(\beta_{0}=0\right)$ in $(9)$, the $t$ and $F$ statistics, corresponding to $H_{0}: \gamma=0$ and $H_{1}: \beta_{0}=\gamma=0$, are denoted $\tau_{\mu}(\tau)$ and $\Phi_{1}$ respectively and the corresponding critical values are also given in the previous references. In all cases the critical values given there crucially depend upon the sample size. It should also be noted that the critical values depend upon the 'nuisance' parameters contained in the model and in the DGP. To discuss this more formally, consider the sample variance of $y_{t}$ when it is generated by (8) (i.e. $\rho=1)$

$$
T^{-1} \Sigma y_{t}^{2}=T^{-1} \Sigma\left[\left(\beta_{0}+\beta_{1} / 2\right)^{2} t^{2}+\left(\beta_{1} / 2\right)^{2} t^{4}+S_{t}^{2}+\left(\beta_{0}+\beta_{1} / 2\right) \beta_{1} t^{3}\right.
$$$$
\left.+2\left(\beta_{0}+\beta_{1} / 2\right) t S_{t}+\beta_{1} t^{2} S_{t}\right]
$$

From the distributional results in Park and Phillips (1988), it is known that $T^{-2} \Sigma S_{t}^{2}, T^{-5 / 2} \Sigma t S_{t}$ and $T^{-7 / 2} \Sigma t^{2} S_{t}$ tend to $f(W)$, hence, by taking probability limits in $(10)$, we get

$T^{-1} \Sigma y_{t}^{2} \Rightarrow \beta_{1}^{2} / 20 \mathrm{O}\left(T^{4}\right)+\left(\beta_{0}+\beta_{1} / 2\right) \beta_{1} / 4 \mathrm{O}\left(T^{3}\right)+\beta f(W) \mathrm{O}\left(T^{5 / 2}\right)$

$$
+\left(\beta_{0}+\beta_{1} / 2\right)^{2} / 3 \mathrm{O}\left(T^{2}\right)+2\left(\beta_{0}+\beta_{1} / 2\right) f(W) \mathrm{O}\left(T^{3 / 2}\right)+f(W) \mathrm{O}(T)
$$

whereby it is seen that

$$
\begin{array}{ll}
T^{-5} \Sigma y_{t}^{2} \Rightarrow \beta_{1}^{2} / 20 & \text { if } \beta_{1} \neq 0 \\
T^{-3} \Sigma y_{t}^{2} \Rightarrow \beta_{0}^{2} / 3 & \text { if } \beta_{0} \neq 0, \beta_{1}=0 \\
T^{-2} \Sigma y_{t}^{2} \Rightarrow f(W) & \text { if } \beta_{0}=\beta_{1}=0
\end{array}
$$

That is, if the unit root process contains a linear trend or a drift, its variability will be dominated by a quadratic or a linear trend which, appropriately normalised, converges to constants. It is only when $\beta_{0}=\beta_{1}=0$ that it converges to a non-standard distribution. This means that for example, if $\beta_{1} \neq 0$ in (8) and the model is estimated as in (9), the quadratic term in (10) will dominate the integrated process and normality of the $\tau_{\tau}$ will follow. Similarly if $\beta_{0} \neq 0$ in (8) (with $\beta_{1}=0$ ) and only a constant appears in (9), the linear trend will dominate and normality of $\tau_{\mu}$ will also follow. ${ }^{7}$ It is only when $\beta_{0}=\beta_{1}=0$, both in the DGP and in the model, that the non-standard distribution will dominate. This implies that in order to use the DF critical values if a linear trend is included in the maintained hypothesis (9), the relevant null hypothesis should be a random walk with drift $\left(\beta_{0} \neq 0\right)$, whilst if only a drift is included in (9), the relevant null hypothesis is a random walk without drift. $^{8}$

From the previous discussion we consider that the following testing strategy is most appropriate. First, start by the most unrestricted model (9), $\left(\beta_{0} \neq 0, \beta_{1} \neq 0\right)$ if it is suspected that the differenced series has a drift. Then use $\tau_{\tau}$ to test for the null hypothesis. If it is rejected there is no need to go further. $\tau_{r}$ to test for the null hypothesis. If it is rejected there is no need to go further.
If it is not rejected, test for the significance of the trend under the null. If it is If it is not rejected, test for the significance of the trend under the null. If it is
significant, then test again for a unit root using the standardised normal. If the trend is not significant in the maintained model, estimate (9) without trend $\left(\beta_{1}=0\right)$. Test again for the unit root using $\tau_{\mu}$. If the null hypothesis is rejected, again there is no need to go further. If it is not rejected, test for the significance of the constant under the null hypothesis and so on.

(ii) In the analysis of the DF tests, we have assumed that the DGP is a pure $\operatorname{AR}(1)$ process. If instead, the DGP is $\operatorname{AR}(p)$

$$
y_{t}=\beta_{0}+\beta_{1} t+\sum_{i=1}^{p} \rho_{i} y_{t-i}+\varepsilon_{t}
$$

let

$$
\lambda^{p}-\sum_{i=1}^{p} \rho_{i} \lambda^{p-i}=0
$$

be the characteristic equation of the time series, where $\lambda(i=1, \ldots, p)$ are the eigenvalues of the process. Dickey and Fuller $(1979,1981)$ consider the problem of testing the null hypothesis $H_{0}: \lambda_{1}=1$ and $\left|\lambda_{2}\right|<1$ for $i=2, \ldots, p$, suggesting OLS estimation of the reparameterised regression model

$$
\Delta y_{t}=\beta_{0}+\beta_{1} t+\gamma_{1} y_{t-1}+\sum_{i=1}^{p-1} \gamma_{2 i} \Delta y_{t-i}+\varepsilon_{t}
$$

where $p$ is large enough to ensure that the residual series $\varepsilon_{t}$ is white noise. The tests are based on the $t$-ratio on $\hat{\gamma}_{1}$ and are known as 'Augmented Dickey Fuller' (ADF) statistics. The critical values are the same as those discussed for the DF statistics, since the $\hat{\gamma}_{2 i}(i=1, \ldots, p-1)$ estimates converge to their true values at statistics, since the $\hat{\gamma}_{2 i}(i=1, \ldots, p-1)$ estimates converge to their true values at
a rate $\mathrm{O}\left(T^{-1 / 2}\right)$, being asymptotically dominated by the distribution of $\hat{\gamma}_{1}$ which, as we mentioned in (4), is $O\left(T^{-1}\right)$. The same testing strategy discussed above, applies in this case. ${ }^{9}$

The sample distribution of the ADF statistics critically depend on the assumption that the time series $y_{t}$ is generated by a pure AR process. However, 
since there is evidence that many macroeconomic series contain moving average (MA) components (see Schwert, 1987), we would want to consider also the possibility of an MA component in the DGP, so that the null hypothesis would be that the data are generated by a mixed autoregressive integrated moving average (ARIMA) process.

Said and Dickey (1984) extend the ADF test by exploiting the fact that an ARIMA $(p, 1, q)$ process can be adequately approximated by a high-order autoregressive process, $\operatorname{AR}(l)$, where $l=\mathrm{O}\left(T^{1 / 3}\right)$ as $T \uparrow \infty$. In practice the test proceeds as before with $p$ in (12) and (14) equal to $l$. This approach permits one to test the null hypothesis of the presence of a unit root without knowing the orders of $p$ and $q$. However, it involves the estimation of additional nuisance parameters which reduces the effective number of observations due to the need for extra initial conditions.

When $p$ and $q$ are known, Said and Dickey (1985) present a test for the hypothesis that the process is $\operatorname{ARIMA}(p, 1, q)$, i.e.

$$
H_{0}: \phi(L) \Delta y_{t}=\theta(L) \varepsilon_{t}
$$

where $\phi($.$) and \theta($.$) are p$ th and $q$ th order polynomials in the lag operator $L$, versus the alternative hypothesis that it is $\operatorname{ARIMA}(p, 0, q)$

$$
H_{1}: \phi(L)(1-\rho L) y_{t}=\theta(L) \varepsilon_{t}
$$

To perform the test of $\rho=1$, we specify initial estimates of the parameters that are consistent under the null and alternative hypothesis. We next perform a onestep of the Gauss-Newton numerical estimation procedure (see, e.g., Harvey, $1981 \mathrm{p} .17)$. The $t$-statistic associated with $\rho$, after applying the iteration has the limiting distribution of $\tau$, tabulated by Fuller $(1976$, p. 373). Similarly if the series mean $y$ is subtracted from each observation of $y_{t}$ prior to analysis, the $t$-statistic has the limiting distribution of $\tau_{\mu}$.

(iii) An alternative approach, based upon the DF procedure has been presented by Phillips (1987) and Phillips and Perron (1988). While the ADF statistics are based upon the assumption that the disturbance term $\varepsilon_{t}$ is identically and independently distributed, they suggest amending these statistics to allow for weak dependence and heterogeneity in $\varepsilon_{t}$. Under such general conditions, a wide class of DGP's for $\varepsilon_{t}$, such as most finite order ARIMA $(p, o, q)$ models, can be allowed. The procedure consists of computing the DF statistics and then using some non-parametric adjustment of $\tau_{\mu}$ and $\tau_{\tau}$ in order to eliminate the dependence of their limiting distributions on additional nuisance parameters stemming from the ARIMA process followed by the error terms. Their adjusted counterparts are denoted $Z\left(\tau_{\mu}\right)$ and $Z\left(\tau_{\tau}\right)$, respectively.

For the regression model (9), with $\beta_{1}=0$, Phillips and Perron (PP) define

$$
Z\left(\tau_{\mu}\right)=\left(\hat{s} / \hat{s}_{T m}\right) \tau_{\mu}-0.5\left(\hat{s}_{T m}^{2}-\hat{s}^{2}\right) T\left\{s_{T m}^{2} \sum_{2}^{T}\left(y_{t}-\bar{y}_{-1}\right)^{2}\right\}^{-1 / 2}
$$

where $T$ is the sample size and $m$ is the number of estimated autocorrelations;
$\bar{y}_{-1}=(T-1)^{-1} \sum_{2}^{T} y_{t-1}, \hat{s}^{2}$ and $\tau_{\mu}$ are, respectively, the sample variance of the residuals and the $t$-statistic associated with $\gamma$ from the regression (9) (with $\beta_{1}=0$ ); and $s_{T m}^{2}$ is the long-run variance estimated as ${ }^{10}$

$$
\hat{s}_{T m}^{2}=T^{-1} \sum_{t=1}^{T} \varepsilon_{t}^{2}+2 T^{-1} \sum_{s=1}^{l} w_{s m} \sum_{t=s+1}^{T} \hat{\varepsilon}_{t} \hat{\varepsilon}_{t-s}
$$

where $\hat{\varepsilon}$ are the residuals from the regression (9) and where the triangular kernel

$$
w_{s m}=[1-s(m+1)], s=1, \ldots, m
$$

is used to ensure that the estimate of the variance $\hat{s}_{T m}^{2}$ is positive (see Newey and West, 1987)

When $\beta_{1} \neq 0$ in (9), the corresponding statistic is

$$
Z\left(\tau_{\tau}\right)=\left(\tilde{s} / \tilde{s}_{T m}\right) \tau_{\tau}-\left(\tilde{s}_{T m}^{2}-\tilde{s}^{2}\right) T^{3}\left\{4 \tilde{S}_{T m}\left[3 D_{x x}\right]^{1 / 2}\right\}^{-1}
$$

where $\tilde{s}$ and $\tilde{s}_{T m}$ are defined as above, but with the residual $e$ obtained from the estimation of (9) with $\beta_{1} \neq 0 . D_{x x}$ is the determinant of the regressor crossproduct matrix, given by

$$
\begin{aligned}
D_{x x}= & {\left[T^{2}\left(T^{2}-1\right) / 12\right] \Sigma y_{t-1}^{2}-T\left(\Sigma t y_{t-1}\right)^{2} } \\
& +T(T+1) \Sigma t y_{t-1} \Sigma y_{t-1}-[T(T+1)(2 T+1) / 6]\left(\Sigma y_{t-1}\right)^{2}
\end{aligned}
$$

The Phillips and Perron statistics have the same limiting distributions as the corresponding DF and ADF statistics, provided that $m \uparrow \infty$ as $T \uparrow \infty$, such that $m / T^{1 / 4} \uparrow 0$.

(iv) Simulation evidence in Molinas (1986) and Schwert $(1986,1989)$, shows tha the tests proposed by Dickey and Fuller and by Phillips and Perron are affected by the process generating the data in large finite samples. In particular, when the underlying process is ARIMA $(0,1,1)$ with a MA parameter close to one, the $\mathrm{ADF}$ and PP statistics have critical values that are far below the Dickey-Fuller distributions (i.e. these tests will lead to the conclusion that economic data are stationary too frequently). The intuition behind this result is that if the DGP of $y_{t}$ is

$$
\Delta y_{t}=(1-\theta L) \varepsilon_{t}
$$

if $\theta$ is close to one $(1-L)$ will tend to cancel on both sides of (19), giving the impression that $y_{t}$ behaves like a white noise. However, the Said and Dickey (1984) high-order autoregressive $t$ test for the unit root, with a suitable choic of $l$, has size close to its nominal level for all values of the MA parameter. Schwert suggests searching for the correct specification of the ARIMA process before testing for the presence of a unit root in the AR polynomial and provides the relevant critical values for the Said and Dickey (1984), Phillips (1987) and Phillips and Perron (1988) tests based on Monte Carlo experiments.

(v) Hall (1989) proposes a new approach to testing for a unit root in a time series with a moving average component based on an instrumental variable (IV) estimator 


$$
y_{t}=\beta_{0}+\beta_{1} t+\rho y_{t-1}+\theta(L) \varepsilon_{t}
$$

where $\theta(L)$ is again a $q$-th order lag polynomial. Then, the IV estimator for model $(20)$ is defined as follows

$$
\left[\tilde{\beta}_{0}, \tilde{\beta}_{1}, \tilde{\rho}_{1}\right]^{\mathrm{IV}}=\left(\sum_{k} Z_{1 t} X_{\mathrm{i} t}\right)^{-1}\left(\sum_{k}^{T} Z_{1 t} y_{t}\right)
$$

where $Z_{1 t}=\left(1, t, y_{t-k}\right)$ and $X_{1 t}=\left(1, t, y_{t-1}\right), k=(q+1)$ (see Dolado (1989) for the choice of optimal IV in this framework). For the model (20) when $\beta_{1}=0$, the IV estimator is given by

$$
\left[\hat{\beta}_{0}, \hat{\rho}_{2}\right]^{\mathrm{IV} \mathrm{V}^{\prime}}=\left(\sum_{k}^{T} Z_{2 t} X_{2 t}^{\prime}\right)^{-1}\left(\sum_{k}^{T} Z_{2 t} y_{t}\right)
$$

where now $Z_{2 t}=\left(1, y_{t-k}\right), X_{1 t}=\left(1, y_{t-1}\right)$

Let $\tilde{t}\left(\tilde{\rho}_{1}^{\mathrm{IV}}\right)$ and $\hat{t}\left(\hat{\rho}_{2}^{\mathrm{IV}}\right)$ be the $t$-statistics associated with the null hypothesis $\rho=1$ in (19) (with and without trend), then Hall proves that

and

$$
\tilde{\tau}_{\mathrm{IV}}=s_{\varepsilon} \tilde{\tau}\left(\tilde{\rho}_{1}^{\mathrm{IV}}\right) / \tilde{s} \Rightarrow \tau_{\tau}
$$

$$
\hat{\tau}_{\mathrm{IV}}=S_{\varepsilon} \hat{t}\left(\hat{\rho}_{2}^{\mathrm{IV}}\right) / \hat{s} \Rightarrow \tau_{\mu}
$$

where $s_{\varepsilon}^{2}, \hat{s}^{2}$ and $\hat{s}^{2}$ are consistent estimators of the variances of $\varepsilon$ and the longrun variance $e(=\theta(L) \varepsilon)$ obtained as in (16) and (17).

(vi) As it might have been noticed, one important limitation of all of the previous testing procedures is that they are not independent of the nuisance parameters contained in the deterministic component of the time-series process. This limitation has produced an alternative strand in the literature on testing. In this respect, Bhargava (1986) has developed most powerful invariant (MPI) tests for the null hypothesis corresponding to DGP (9) (with and without trend). These tests are valid in small samples and are independent of the nuisance parameters, but only valid for the AR(1) case. They are based upon transformations of Von Neumann type ratios, as for example the Durbin-Watson approach emphasised by Sargan and Bhargava (1983) in a different context, as discussed below. The statistics proposed to test $H_{0}: \rho=1$, when $\beta_{1}=0$ and $\beta_{1} \neq 0$, are given by

$$
R_{1}=\sum_{2}^{T}\left(\Delta y_{t}\right)^{2} / \sum_{2}^{T}\left(y_{t}-\bar{y}\right)^{2}
$$

and

$$
R_{2}=\left[\sum_{2}^{T}\left(\Delta y_{t}\right)^{2}-(T-1)^{-1} \sum_{1}^{T} \Delta y_{i}\right] / D
$$

where

$D=(T-1)^{-2} \sum_{1}^{T}\left[(T-1) y_{t}-(t-1) y_{T}-(T-t) y_{1}-(T-1)\left(\bar{y}-0.5\left(y_{1}+y_{T}\right)\right)\right]^{2}$

The corresponding critical values are given by Bhargava $(1986, p .378)$. The test is found to have slightly greater power than the tests proposed by Dickey and Fuller, when the data are generated by an AR(1) process.

(vii) Another limitation of all the previous testing procedures is that the distributions of the corresponding statistics are non-standard and hence a different set of critical values has to be used in each case. This problem has originated a new strand of research (see Phillips and Ouliaris, 1988), which exploits the fact that differencing a stationary series induces a unit root in the moving average representation. This fact provides a diagnostic for testing whether the series is $I(0)$ or $I(1)$, by using the long-run variance of the first difference of the time series $y_{t}$. To clarify the interpretation of the test, let us assume that $y_{t}$ is generated by

$$
\Delta y_{t}=\theta(L) \varepsilon_{t} ; \theta(L)=\left(1-\theta_{1} L\right) \theta^{\prime}(L)
$$

Then the long-run variance of $\Delta y_{t}$ is $\sigma^{2}=\sigma_{\varepsilon}^{2} \theta(1)^{2}$. If $\theta_{1} \neq 1$ and $\theta^{\prime}(1) \neq 0$, then $\sigma^{2}$ is finite, whilst if $\theta_{1}=1, \sigma^{2}$ is zero. In other words, if the time series $y_{t}$ is $I(0)$, $\Delta y_{t}$ will have $\sigma^{2}=0$, whereas if it is $I(1), \sigma^{2} \neq 0$. Therefore the null hypothesis is $H_{0}: \sigma^{2} \neq 0$ or $H_{0}: \tau^{2}=\sigma^{2} / \sigma_{\varepsilon}^{2} \neq 0$, getting rid of the units of measurement. Obtaining an estimate of $\sigma^{2}$ as in (16), Phillips and Ouliaris prove that

$$
m^{1 / 2}\left(\hat{\tau}^{2}-\tau^{2}\right) / \tau^{2} \sim N(0,1)
$$

Since only the alternative hypothesis is a simple hypothesis, i.e. $H_{1}: \tau^{2}=0$, Phillips and Ouliaris propose a bounds procedure based upon the corresponding confidence interval in (27), yielding

$$
\hat{\tau}^{2} /\left[1+\left(z_{\alpha} / m^{1 / 2}\right)\right] \leqslant \tau^{2} \leqslant \hat{\tau}^{2} /\left[1-\left(z_{\alpha} / m^{1 / 2}\right)\right]
$$

where $z_{\alpha}$ is the $(1-\alpha)$ percentage point of the standard normal distribution. According to the bounds test, $H_{0}$ is rejected if the upper limit of $\tau^{2}$ in (28) is sufficiently small. Similarly $H_{0}$ is not rejected if the lower bound is sufficiently large. Phillips and Ouliaris recommend using 0.10 as the rejection point for the upper and lower bound. Simulation results show, however, that the suggested value can be very conservative in some instances. For example if the DGP is ARIMA $(1,1,1)$ with parameter values in the interval $(-0.6,0.6)$, the average upper bound is 0.45 whereas the value of the lower bound is close to 0.10 .

A very nice implication of this type of tests is that, given their asymptotic normality, they can be applied to deal with very general trend-cycle models (e.g. piecewise linear functions of time, any type of impulse or step dummy). All that is needed is to perform the previous test on the differenced residuals of the regression of $y_{t}$ on the general trend function. 


\subsection{Integration and seasonality}

Due to the fact that many economic time series contain important seasonal components, there have been several developments in the concept of seasonal integration.

Osborn et al. (1988) amend the Engle and Granger (1987) definition of integration to account for seasonality: a variable $y_{t}$, is said to be integrated of order $(d, D)$ [or $y_{t} \sim I(d, D)$ ], if it has a stationary, invertible, non-deterministic ARMA representation after one-period differencing $d$ times and seasonally differencing $D$ times.

Following Pierce (1976), let us assume that seasonality has both deterministic and stochastic components, then a seasonal observed series $y_{t}$ can be seen as the sum of a purely stochastic process $x_{t}$ and a purely deterministic seasonal component $\mu_{t}$

$$
y_{t}=x_{t}+\mu_{t}
$$

where

$$
\mu_{t}=\beta_{0}+\beta_{1} t+\sum_{j=1}^{q-1} \beta_{2 j} S_{j t}
$$

where $S_{j t}$ are zero/one seasonal dummies, and $q=12$ for monthly data, $q=4$ for quarterly data and so on.

By regressing $y_{t}$ on $\mu_{t}$, we can remove the deterministic seasonality, using the residuals from that regression as if they were the true $x_{t}$. Then, the following tests can be applied for testing $I(d, D)$ integration, where we present the case for $q=4$ (i.e. we are dealing with quarterly data).

(i) Dickey et al. (1984) present a test for the presence of a single unit root at a seasonal lag. The null hypothesis is $H_{0}: I(0,1)$ and the alternative is $H_{1}: I(0,0)$. The test is a 3-step procedure as follows:

1. The regression equation

$$
\Delta_{4} x_{t}=\theta_{0}+\sum_{i=1}^{p} \theta_{i} \Delta_{4} x_{t-i}+\varepsilon_{t}
$$

is estimated by OLS, where $\Delta_{4} x_{t}=x_{t}-x_{t-4}$

2. Using the estimates $\hat{\theta}_{1}, \hat{\theta}_{2}, \ldots, \hat{\theta}_{p}$ define

$$
z_{t}=\hat{\theta}(L) x_{t}=\left(1-\hat{\theta}_{1} L-\cdots-\hat{\theta}_{p} L^{p}\right) x_{t}
$$

3. Run the regression

$$
\Delta_{4} x_{t}=\xi_{0} z_{t-1}+\sum_{i=1}^{p} \xi_{i} \Delta_{4} x_{t-i}+\varepsilon_{t}
$$

and compute the $t$-ratio on $\hat{\xi}_{0}$. This sample statistic, denoted $\tau_{\mu 4}$ is compared to the tabulated critical values given in Table 7 of Dickey, Hasza and Fuller (1984), p. 362). (ii) Dickey et al. (1986, Appendix B) show that the limiting distribution of the (ii) Dicket statistics is not affected by removal of seasonal means from unit root statistics. Therefore, we can use the ADF statistic from the regression autoregressi

$$
\Delta x_{t}=\gamma_{1} \Delta x_{t-1}+\sum_{i=1}^{p} \gamma_{2 i} \Delta x_{t-i}+\varepsilon_{t}
$$

to test the null hypothesis $H_{0}: x_{t} \sim I(1,0)$ versus the alternative $H_{1}: x_{t} \sim I(0,0)$ The relevant critical values are given by Fuller $\left(1976\right.$, p. 373) for $\tau_{\mu}$.

(iii) Engle et al. (1987) present the following 3-step procedure to test for seasonal unit roots in the possible presence of a zero frequency unit root:

1. Compute $\hat{\theta}(L)$ as for the Dickey et al. (1984) statistic

2. Compute

$$
\begin{aligned}
& z_{1 t}=\hat{\theta}(L)\left(1+L+L^{2}+L^{3}\right) x_{t} \\
& z_{2 t}=-\hat{\theta}(L)\left(1-L+L^{2}-L^{3}\right) x_{t} \\
& z_{3 t}=-\hat{\theta}(L)\left(1-L^{2}\right) x_{t}
\end{aligned}
$$

3. Run the regression

$$
\Delta_{4} x_{t}=\pi_{1} z_{1 t-1}+\pi_{2} z_{2 t-1}+\pi_{3} z_{3 t-2}+\sum_{i=1}^{p} \pi_{4 i} \Delta_{4} x_{t-i}+\varepsilon_{t}
$$

and compute the values of the $t$-ratios on $\hat{\pi}_{1}, \hat{\pi}_{2}$ and $\hat{\pi}_{3}$. The critical values are given in Table 2.1 of Engle et al. (1987, p. 14). If $x_{t} \sim I(0,0)$, then all three of these statistics should be significant. If the test statistic for $\pi_{1}=0$ is not significant, then $x_{t} \sim I(1,0)$. If either of the test statistics for $\pi_{2}=0$ or $\pi_{3}=0$ is not significant, then $x_{t} \sim I(0,1)$

(iv) Osborn et al. (1988) present an alternative 3-step test procedure:

1. Run the regression

$$
\Delta \Delta_{4} x_{t}=\psi_{0}+\sum_{i=1}^{p} \psi_{i} \Delta \Delta_{4} x_{t-i}+\varepsilon_{t}
$$

and compute $\bar{\psi}_{1}, \hat{\psi}_{2}, \ldots, \hat{\psi}_{p}$

2. Compute $z_{4 t}=\hat{\psi}(L) \Delta_{4} x_{t}$ and $z_{5 t}=\bar{\psi}(L) \Delta x_{t}$, where

$$
\hat{\psi}(L)=\left(1-\bar{\psi}_{1} L-\cdots-\hat{\psi}_{p} L^{p}\right)
$$

3. Run the regression

$$
\Delta \Delta_{4} x_{t}=\phi_{1} z_{4 t-1}+\phi_{2} z_{5 t-4}+\sum_{i=1}^{p} \phi_{3 i} \Delta \Delta_{4} x_{t-i}+\varepsilon_{t}
$$

and compute the $F$-statistics for the null hypothesis $H_{0}: \phi_{1}=\phi_{2}=0$, and the $t$-ratio on $\phi_{1}$ and $\phi_{2}$. The null hypothesis for both type of statistics is $H_{0}: x_{t} \sim I(1,1)$ with alternative hypothesis $H_{1}: x_{t} \sim I(0,0)$ or $H_{2}: x_{t} \sim I(0,1)$. The 
critical values of these statistics are given in Table A.1 of Osborn et al. (1988, p. 376).

\section{Other forms of integration}

In this section, we review alternative forms of integration based upon the possibility that the model parameters are allowed to vary (periodic integration) or the possibility of using non-integer differencing orders to achieve stationarity in the data (fractional integration). Both ideas have received recent attention in the literature.

\subsection{Periodic Integration}

Osborn et al. (1988), building upon the framework developed by Tiao and Grupe (1980), investigate the use of a periodic model (whose parameters are allowed to vary according to the time at which observations are made) as an alternative to the conventional approaches to modelling for seasonal data.

The non-deterministic periodic AR(1) process is given by the following expression

$$
y_{t}=\sum_{j=1}^{q} \omega_{j} S_{j t} y_{t-1}+\varepsilon_{t}
$$

or

$$
y_{t}=\omega_{j} y_{t-1}+\varepsilon_{t}
$$

when $t$ falls in season $j$. As in equation (30), $S_{j t}$ are seasonal dummy variables corresponding to season $j(j=1, \ldots, q)$. Equation (33) states that $y_{t}$ is seasonal, seasonality arising not from any direct dependence of $y_{t}$ on $y_{t-q}$, but from the annual variation in the autoregressive coefficients $\omega_{j}$. This dependence can arise, for example, if the allocation of expenditure over the year reflects seasonal tastes and hence seasonality in the underlying utility function (see Osborn, 1988).

Osborn et al. (1988) define periodic integration as follows: A variable $y_{t}$ is periodically integrated of order one [or $\left.y_{t} \sim \mathrm{PI}(1)\right]$ if $y_{t}$ is non-stationary and $\delta_{j} y_{t}$ is stationary, where the generalised difference operator $\delta_{j}$ is defined as

$$
\delta_{j} y_{t}=y_{t}-\omega_{j} y_{t-1}
$$

the product $\omega_{1}, \omega_{2}, \ldots, \omega_{q}$ being equal to one

Osborn et al. (1988) propose two ways of testing for periodic integration:

(i) After regressing $y_{t}$ on $\mu_{t}$ (as defined in (30)) to remove conventional deterministic seasonality, a non-deterministic periodic AR(1) process (as defined in (33)) is fitted to the residuals $x_{t}$. This case is referred to as the removed deterministic seasonality case.

(ii) The case of included deterministic seasonality is given by fitting the following periodic AR(1) process to the original observations $y_{t}$

$$
y_{t}=\Omega_{j}+\omega_{j} y_{t-i}+\varepsilon_{t}(j=1, \ldots, q)
$$

To allow for the possibility of a periodic disturbance variance, they suggest a 2-step estimation procedure for both cases. In the first step, the appropriate equation is estimated by OLS applied to observations on each of the $q$ seasonal realisations, (i.e. four for quarterly data); then the equation is transformed by dividing each variable by the appropriate seasonal residual standard deviation estimated in this first stage regression. Using the transformed data, in the second step the periodic AR(1) model is estimated in its two versions (i.e. removed and included deterministic seasonality), with imposition of the restriction $\omega_{1}, \omega_{2}, \ldots, \omega_{q}=1$.

Finally, the tests (i) to (iii) in Section 3.2 are applied to the residuals of the periodic AR(1) model.

\subsection{Fractional integration}

As was seen in Section 2, one of the main characteristics of the existence of unit roots in the Wold representation of a time series is that they have 'long memory' (i.e. shocks have a permanent effect on the level of the series). In general it is known that the coefficient on $\varepsilon_{t-j}$ in the MA representation of any $I(d)$ process has a leading term $j^{d-1}$ (for example, the coefficient in a random walk is unity since $d=1$ ). This implies that the variance of the original series is $\mathrm{O}\left(t^{2 d-1}\right)$. So, all that is needed to have 'long-memory', in the sense that the variance explodes as $t \uparrow \infty$, is a degree of differencing $|d|>0.5$. Thus, it is clear that a wide range of dynamic behaviour is ruled out a priori if $d$ is restricted to integer values.

Granger and Joyeux (1980) and more recently Diebold and Rudebusch (1989) have proposed a new family of "long-memory" processes, denoted by ARFIMA (autoregressive fractionally integrated moving-average processes), of which the ARIMA processes are particular cases: A variable $y_{t}$ is fractionally integrated of order $d\left[\right.$ or $\left.y_{t} \sim \mathrm{FI}(d)\right]$ if $y_{t}$ is non-stationary and $\Delta^{d}$ is stationary, where the order $d\left[\right.$ or $\left.y_{t} \sim \operatorname{FI}(d)\right]$ if $y_{t}$ is non-stationary and $\Delta^{d}$ is
operation $\Delta^{d}$, using a binomial expansion, is as follows

$$
(1-L)^{d}=1-d L+\frac{d(d-1)}{2 !} L^{2}-\frac{d(d-1)(d-2)}{3 !} L^{3}+\cdots
$$

where $d$ belongs to the rational set of numbers and $d>0.5$.

Note that these processes can always be constrained to belong to the open interval $(0.5,1.0)$ by subtracting the integer part of the differencing order. So if the degree of differencing is, for example, 1.7 , we can always redefine the degree of differencing as $d-1$ ( 0.7 in this case).

Diebold and Rudebusch (1989) propose the following method of testing and estimation for fractional integration:

(i) First difference the relevant series denoted $\tilde{y}_{t}=(1-L) y_{t}$. As $d$ of the level series equals $1+\tilde{d}$, a value of $\tilde{d}$ equal to zero corresponds to a unit root in $y_{t}$. 
Thus, we wish to estimate $\tilde{d}$ in the model

$$
(1-L)^{d} \tilde{y}_{t}=\theta(L) \varepsilon_{t}
$$

(ii) Estimate by OLS the following regression

$$
\ln \left[I\left(\lambda_{j}\right)\right]=\beta_{0}-\beta_{1} \ln \left\{4 \sin ^{2}\left(\lambda_{j} / 2\right)\right\}+\eta_{j}, j=1, \ldots, T^{1 / 2}
$$

where $\lambda_{j}=2 \pi_{j} \mid T(j=0, \ldots, T-1)$ denote the harmonic ordinates of the sample and $I\left(\lambda_{j}\right)$ denote the periodogram at ordinate $j$ (see Harvey, 1981, p. 66) Geweke and Porter-Hudak (1983) prove that $\hat{\beta}_{1}$ is a consistent and asymptotically normal estimate of $d$. Furthermore, the variance of the estimate of $\beta_{1}$ is given by the usual OLS estimator, which can be used to test the null hypothesis $H_{0}: d=0$ [i.e. $y_{t} \sim I(1)$ ]. Moreover they show that the variance of the disturbance $\eta_{j}$ is known to be equal to $\pi^{2} / 6$, which can be imposed to increase efficiency.

(iii) Given an estimate of $\tilde{d}$ we transform the series $\tilde{y}_{t}$ by the 'long-memory' filter (35), truncated at each point to the available sample. The transformed series is then modelled as in (36) (or in the ARMA representation) following the traditional Box and Jenkins (1970) procedure.

\section{Testing for stationarity in the cointegrating residuals}

In the two previous sections we have discussed procedures to test for the order of integration of individual time series. This is, as we mentioned in Section 2, a first stage in the estimation and testing of cointegrating relationships. The reason is a matter of 'integration or growth accounting' in the words of Pagan and Wickens (1989) (i.e. the left and right hand sides of an equation, such as (4) must be of the same order of integration, otherwise, the residual will not be stationary). If for example, the dependent variable is $I(1)$, the independent variables need to be $I(1)$ and not cointegrate among themselves to an $I(0)$ variable or, perhaps, be $I(2)$ and cointegrate among themselves to an $I(1)$ variable.

In order to illustrate testing for cointegration, we will consider a bivariate case where say, $y_{t}$ and $x_{t}$ have been found to contain a single unit root at the regular frequency (i.e. both are $I(1)$ ). Then, the following part of the cointegration test is to estimate the cointegrating regression (4) and test whether the 'cointegrating residuals' $\left(\hat{z}_{t}=y_{t}-\hat{\alpha}-\hat{\beta} x_{t}\right)$ are $I(0)$.

Engle and Granger (1987) suggest seven alternative tests for determining if $z$ is stationary. Here we will consider only two of their suggested tests, namely the Durbin-Watson statistic for the cointegration equation (CRDW) and the ADF statistic for the cointegrating residuals (CRADF).

The DW statistic for equation (4) will approach zero if the cointegrating residuals contain an autoregressive unit root, and thus the test rejects the nul hypothesis of non-cointegration if the CRDW is significantly greater than zero. The intuition underlying this test can be understood by means of a simple example. Suppose that $z_{t}$ is assumed to follow an $\operatorname{AR}(1)$ process with coefficient $\rho$. Then the null hypothesis of non-cointegration is $H_{0}: \rho=1$. Since it can be shown that the DW statistic is such that $\mathrm{DW} \simeq 2(1-\rho)$ (see, e.g. Harvey, 1981 , p. 20), the previous null hypothesis can be translated into $H_{0}: \mathrm{DW}=0$ versus the alternative $H_{1}: \mathrm{DW}>0$. Engle and Granger $(1987$, p. 269$)$ present the critical values of this test for 100 observations.

The CRADF statistic is based upon the OLS estimation of

$$
\Delta \hat{z}_{t}=\gamma_{1} \hat{z}_{t-1}+\sum_{i=1}^{p} \gamma_{2 i} \Delta \hat{z}_{t-i}+\varepsilon_{t}
$$

where again $p$ is selected on the basis of being sufficiently large to ensure that $\varepsilon_{t}$ is a close approximation to white noise. The $t$-ratio statistic on $\hat{\gamma}_{1}$ is the CRADF statistic. We cannot use the critical values tabulated by Fuller (1976) to test for a unit root in the cointegrating residuals. Intuitively, since OLS estimation of the cointegrating regression equation chooses $\alpha$ and $\beta$ to minimise the residual variance, we might expect to reject the null hypothesis $H_{0}$ : $z_{t} \sim I(1)$ rather more often than suggested by the nominal test size, so that the critical values have to be raised in order to correct the test bias. Engle and Granger (1987, p. 269) present the critical values for the CRADF statistic generated from Monte Carlo simulations of 100 observations.

generated from Monte Carlo simulations of 100 observations. bivariate case (i.e., for one dependent and one independent variable in the cointegrating regression), and for 100 observations. Engle and Yoo (1987) produce expanded critical values for CRDW and CRADF statistics for 50, 100 and 200 observations, and for systems of up to five variables.

\section{Some new developments in cointegration}

In this section we survey some new test procedures for cointegration that have recently been proposed in the literature. Most of these procedures extend the testing and estimation approach introduced in Section 2 to a multivariate context where there may exist more than a single cointegrating relationship among a set of $n$ variables. For example, among nominal wages, prices employment and productivity, there may exist two relationships, one determining employment prod

In general, if $X_{t}$ represents a vector of $n I(1)$ variables whose Wold representation is

$$
\Delta X_{t}=C(L) \varepsilon_{t}
$$

where now $\varepsilon_{t} \sim \operatorname{nid}(0, \Sigma), \Sigma$ being the covariance matrix of $\varepsilon_{t}$ and $C(L)$ an invertible matrix of polynomial lags. If there exists a cointegrating vector $\alpha$, then, premultiplying (39) by $\alpha^{\prime}$, we obtain

$$
\alpha^{\prime} \Delta X_{t}=\alpha^{\prime}\left[C(1)+C^{*}(L)(1-L)\right] \varepsilon_{t}
$$

where $C(L)$ has been expanded around $L=1$ and $C^{*}(L)$ can be shown to be invertible (see Engle and Granger, 1987). If the linear combination $\alpha^{\prime} X_{t}$ is 
stationary, then $\alpha^{\prime} C(1)=0$ and then $(1-L)$ would cancel out on both sides of (40). If (39) is represented in AR form, we have that

$$
A(L) C(L)=(1-L) I
$$

where $I$ is an identity matrix, and hence

$$
A(1) C(1)=0
$$

This implies that $A(1)$ can be written as $A(1)=\gamma \alpha^{\prime}$. If there were $r$ cointegrating vectors $(r \leqslant n-1)$, then $A(1)=B \Gamma^{\prime}$, where $B$ and $\Gamma$ are $(n \times r)$ matrices which collect the $r$ different $\gamma$ and $\alpha$ vectors. Testing the rank of $A(1)$ or $C(1)$ constitutes the basis of the following procedures:

(i) Johansen (1988) and Johansen and Juselius (1988) develop a maximum likelihood estimation procedure that has several advantages on the 2-step regression procedure suggested by Engle and Granger. It relaxes the assumption that the cointegrating vector is unique and it takes into account the error structure of the underlying process.

Johansen considers the $p$-th order autoregressive representation of $X_{t}$

$$
X_{t}=\Pi_{1} X_{t-1}+\Pi_{2} X_{t-2}+\cdots+\Pi_{p} X_{t-p}+\varepsilon_{t}
$$

which, following a similar procedure to the $\mathrm{ADF}$ test, can be reparameterised as

$$
\Delta X_{t}=\tilde{\Pi}_{i} \Delta X_{t-1}+\cdots+\tilde{\Pi}_{p-1}^{\prime} \Delta X_{t-p+1}+\tilde{\Pi}_{p}^{\prime} X_{t-p}+\varepsilon_{t}
$$

where $\tilde{\Pi}_{p}^{\prime}=-\Pi(1) \quad\left(=-\left(\Pi_{1}+\cdots+\Pi_{p}\right)\right)$. To estimate $\tilde{\Pi}_{p}^{\prime}$ by maximumlikelihood, we estimate by OLS the following regressions

and

$$
\Delta X_{t}=\Gamma_{01} \Delta X_{t-1}+\cdots+\Gamma_{0 k-1} \Delta X_{t-k+1}+e_{0 t}
$$

$$
X_{t-p}=\Gamma_{11} \Delta X_{t-1}+\cdots+\Gamma_{1 k-1} \Delta X_{t-k+1}+e_{1 t}
$$

and compute the product moment matrices of the residuals

$$
\hat{S}_{i j}=T^{-1} \sum_{i=1}^{T} \hat{e}_{i t} \hat{e}_{j t} ; i, j=0,1
$$

The likelihood ratio test statistic of the null hypothesis $H_{0}: \Pi_{p}^{\prime}=\mathrm{B} \Gamma^{\prime}$, i.e. there are at most $r$ cointegrating vectors, is

$$
-2 \ln (Q)=-T \sum_{i=r+1}^{p}\left(1-\hat{\lambda}_{i}\right)
$$

where $\hat{\lambda}_{r+1}, \ldots, \hat{\lambda}_{p}$ are the $p-r$ smallest eigenvalues of $\hat{S}_{10} \hat{S}_{00} \hat{S}_{01}$ with respect to $S_{11}$, obtained from the determinant

$$
\left|\hat{\lambda} \hat{S}_{11}-\hat{S}_{10} \hat{S}_{00} \hat{S}_{01}\right|=0
$$

Under the hypothesis that there are at most $r$ cointegrating vectors, Johansen (1988) shows that the likelihood ratio test (45) is asymptotically distributed as a functional $f(W)$. Johansen $(1988$, p. 239) provides a table with various quantiles of the distribution of the likelihood ratio test for $r=1,2, \ldots, 5$. He also shows that these quantiles can be obtained by approximating the distribution by $c \chi^{2}(f)$ where $c=0.85-0.85 / f$, and $\chi^{2}(f)$ is a central chi-square distribution with $f=2(p-r)^{2}$ degrees of freedom

(ii) Stock and Watson (1988) focus on testing for the rank of $C(1)$ in (40) and denote their approach as a 'common trends' approach, by noticing that if there exist $r$ cointegrating vectors in (40), then there exists a representation such that ${ }^{11}$

$$
x_{t}=C(1) \Phi \tau_{t}+C^{*}(L) \varepsilon_{t}
$$

where $\Phi$ is an $n \times(n-r)$ matrix and $\tau_{t}$ is an $n-r$ vector random walk. In other words, $x_{t}$ can be written as the sum of $n-r$ common trends and an $I(0)$ component. Estimating (39) as a multivariate $\operatorname{ARMA}(1, q)$ model, the null hypothesis that there are $r$ cointegrating vectors is equivalent to the null hypothesis that there are $n-r$ 'common trends'. This implies that, under the null hypothesis, the first $(n-r)$ eigenvalues of the autoregressive matrix should be unity and the remaining eigenvalues should be smaller than one. The test is based on $T\left(\hat{\lambda}_{n-r+1}-1\right)$ and the critical values can be found in Stock and Watson on $T\left(\widehat{\lambda}_{n-r+1}-1\right)$
$(1988$, p. 1104).

Phillips and Ouliaris (1988) have also proposed a multivariate extension of their unit root test, as discussed in Section 3, based upon the eigenvalues of the long-run variance of the differenced multivariate series.

(iii) As discussed in Section 2, when concentrating on a single equation estimator in the case of a single cointegrating $C(1,1)$ relationship, the OLS estimator of the slope in the static regression (4) is 'super-consistent' but its distribution is, in general, non-normal and in finite samples is biased (see Banerjee et al., 1986 and Gonzalo, 1989).

This bias and non-normality stem from the $I(1)$ character of the regressor and its possible correlation with the $I(0)$ disturbance $z_{t}$. Phillips (1988) has shown that in the case where $x_{t}$ and $z_{t}$ are independent at all leads and lags, the distribution is a 'mixture of normals' and, hence, the distribution of the $t$-statistic on $\beta$ is asymptotically normal. Phillips and Hansen (1988) have developed an estimation procedure, equivalent to FIML, which corrects for the bias and yields asymptotic normality in the case where such correlation exists. The procedure, denoted as a 'fully modified estimator' (FME), is based upon a 'non-parametric' correction by which the error term $z_{t}$ is conditioned on the process followed by $\Delta x_{t}$ and, hence, orthogonality between regressors and disturbance is achieved by construction. The FME estimators of $\alpha$ and $\beta$ in (4) are given by

$$
(\hat{\alpha}, \hat{\beta})^{+\prime}=\left(\sum_{t=1}^{T} X_{t} X_{t}^{\prime}\right)^{-1}\left[\sum_{t=1}^{T} X_{t}\left(y_{t}-\hat{\sigma}^{2} \Delta x_{t}\right)-e_{2} T\left(\hat{\Delta}_{21}-\hat{\sigma}^{2} \hat{\sigma}_{\Delta x}^{2}\right)\right]
$$


where $X_{t}=\left(1, x_{t}\right), e_{2}=(0,1)^{\prime}$ and

$$
\begin{aligned}
\hat{\Delta}_{21} & =T^{-1} \sum_{k=0}^{l} \sum_{t=k+1}^{T} \Delta x_{t-k} \hat{z}_{t} \\
\hat{\sigma}^{2} & =\hat{\sigma}_{\Delta x, z} / \hat{\sigma}_{\Delta x}^{2}
\end{aligned}
$$

the long-run variances obtained from the first-stage residuals $\hat{z}_{t}$, as in Engle and Granger (1987). Notice that when $\sigma^{2}=\Delta_{21}=0$ the FME estimators coincide with OLS for the static regression (4).

It is interesting to notice that the FME procedure coincides with the Hendry-Sargan approach, as summarised in (3), through the ECM representation of dynamic single equation models, except when $z_{t}$ or $\Delta x_{t}$ contain a moving-average disturbance in their respective representations. Even in that case it is possible to modify slightly equations like (3) by including leads of $\Delta x_{t}$ in the regression model (see Saikkonen, 1989).

\section{Brief conclusion}

The considerable gap between the economic theorist, who has much to say about equilibrium but relatively little to say about dynamics, and the econometrician, whose models concentrate on dynamic adjustment process, has, to some extent, been bridged by the concept of cointegration. In addition to allowing the data to determine the dynamics of the model, cointegration suggests that models can be significantly improved by introducing, and allowing the data to parameterise, equilibrium conditions suggested by economic theory. Furthermore, the generic existence of such long-run relationship can, and should, be tested, using the battery of tests for unit roots discussed in this paper.

\section{Acknowledgements}

We are grateful to A. Banerjee, P. Burridge, A. Escribano, A. Espasa, J. Galbraith, D. Hendry and A. Maravall and an anonymous referee for their comments on previous versions of this paper. The usual caveat applies.

\section{Notes}

1. There is an early survey by two of us (see Dolado and Jenkinson, 1987) and a more recent one by one of us (see Sosvilla-Rivero, 1989), and some excellent overviews by Granger (1986), Hendry (1986), Gilbert (1986), Stock and Watson (1987), Diebold Even though (1988), Pagan and Wickens (1989) and Haldrup and Hylleberg (1989) explicit causal relationship. Here we have assumed that the causal res not imply any by the theory (i.e. $x_{t}$ causes $y_{t}$ ) is the correct one. See Granger (1988) for a study of cointegration and causality.

3. Nickell (1985) shows that the ECM is also consistent with optimising behaviour on the part of economic agents.
4. Alternatively we would say that a 'super-consistent' estimator is such that $\hat{\beta}-\beta$ has

(he 'integration in variance'. Integration pan a lso be applied to other higher moments (see Escribano (1987) and Hansen 88)).

6. See the Appendix for a description for the sequential test procedure $k(k \geqslant 2)$ unit roots. Note that the alternative sequence of testing for the presence of a unit root in the series levels and if it is not rejected, then test for a second unit root, i.e. a unit root in the differences, and so on is not well founded on statistical grounds since the unit root tests described in Section 3 are based on the assumption of stationarity under the alternative hypothesis.

7. This result has been noticed by West (1988) and it is applicable also to regression models like (2) where $x_{t}$ has a unit root with drift. However, Hylleberg and Mizon (1989) have noted in simulation studies that the drift has to be quite large for the deterministic trend to dominate the integrated component. If there are two $I(1)$ regressors with drift in the model, a trend should also be included to avoid asymptotic perfect collinearity.

8. Ouliaris et al. (1988) compute critical values when in the maintained hypothesis there is up to a quintic trend. Similarly, Perron (1987) computes critical values when there is a piecewise linear trend under the maintained hypothesis.

9. Sims et al. (1986) and Banerjee and Dolado (1988), have shown that the estimates of coefficients on $I(0)$ variables in regression models with $I(1)$ variables are $0\left(T^{1 / 2}\right)$ and asymptotically normally distributed.

10. In the frequency domain notation, the long-run variance is equal to $2 \pi f_{c}(0)$, where $f_{\varepsilon}(0)$ in the spectrum of $\varepsilon_{t}$ evaluated at frequency zero.

1. The size of $C(1)$ in a univariate context, has been called the 'size the unit root', giving rise to a literature (see Cochrane (1988) and references therein) which deals with the time-series.

\section{Appendix: testing for $\boldsymbol{k}$ unit roots}

Dickey and Pantula (1987) suggest a sequence of tests for unit roots, starting with the largest number of roots under consideration $(k)$ and decreasing by on each time the null hypothesis is rejected, stopping the procedure when the null hypothesis is accepted.

They illustrate their sequential procedure for the case $k=3$. It is as follows:

1. Run the regression

$$
\Delta^{3} y_{t}=\xi_{0}+\xi_{1} \Delta^{2} y_{t-1}+\varepsilon_{t}
$$

(where $\Delta^{3}$ denotes third difference), and compute the 'pseudo $t$-statistic' $t_{3, n}^{*}(3)$ (i.e. the $t$-statistic on $\hat{\xi}_{1}$ ). Reject the null hypothesis $H_{3}$ of three unit roots and go to step 2 if $t_{3, n}^{*}(3)<\tau_{\mu}$ (or $t_{3, n}^{*}(3)>\tau_{\mu}$ if absolute values are considered) where $\tau_{\mu}$ is given by Fuller ((1976), p. 373).

2. Run the regression

$$
\Delta^{3} y_{t}=\xi_{0}^{\prime}+\xi_{1}^{\prime} \Delta^{2} y_{t-1}+\xi_{2}^{\prime} \Delta y_{t-1}+\varepsilon_{t}
$$

and compute $t_{3, n}^{*}(3)$ and $t_{2, n}^{*}(3)$. Reject the null hypothesis $H_{2}$ of exactly two unit 
roots and go to step 3 if in addition to $t_{3, n}^{*}(3)<\tau_{\mu}$ it is also found that $t_{2, n}^{*}(3)<\tau_{\mu}$ 3. Run the regression

$$
\Delta^{3} y_{t}=\xi_{0}^{*}+\xi_{1}^{*} \Delta^{2} y_{t-1}+\xi_{2}^{*} \Delta y_{t-1}+\xi_{3}^{*} y_{t-1}+\varepsilon_{t}
$$

and compute $t_{3, n}^{*}(3), t_{2, n}^{*}(3)$ and $t_{1, n}^{*}(3)$. Reject the null hypothesis $H_{1}$ of exactly one unit root in favour of the hypothesis $H_{0}$ if $t_{i, n}^{*}(3)<\tau_{\mu}(i=1,2,3)$

\section{References}

Banerjee, A. and Dolado, J. (1988) Tests of the life cycle permanent income hypothesis in the presence of random walks: Asymptotic theory and small-sample interpretations. Oxford Economic Papers 40, 610-633.

Banerjee, A., Dolado, J., Hendry, D. and Smith G. (1986) Exploring equilibrium relationships in econometrics through static models: Some Monte-Carlo evidence. Oxford Bulletin of Economics and Statistics 48, 253-277.

Bhargava, A. (1986) On the theory of testing for unit roots in observed time series. Review of Economic Studies 53, 369-384.

Box, G. and Jenkins, G. (1970) Time Series Analysis, Forecasting and Control. San Francisco: Holden-Day.

Cochrane, J. (1988) How big is the random walk in GNP? Journal of Political Economy

avidson, J., Hendry, D., Srba, F. and Yeo, S. (1978) Econometric modelling of the aggregate time-series relationship between consumers' expenditure and income in the

Dickey, D. and Fuller, W. (1979) Distribution of the estimators for autoregressive timeseries with a unit root. Journal of the American Statistical Association 74, 427-431. (1981) Likelihood ratio statis

Dickey, D. and Pantula, S. (1987) Determining the order of differencing in autoregressive processes. Journal of Business and Economic Statistics 15, 455-461.

Dickey, D., Bell, W. and Miller, R. (1986) Unit roots in time series models: Tests and implications. The American Statistician 40, 12-26.

Dickey, D., Hasza. D. and Fuller, W. (1984) Testing for unit roots in seasonal time series. Journal of the American Statistical Association 79, 355-367.

Diebold, F. and Nerlove, M. (1988) Unit roots in economic time series: A selective survey. Discussion Paper No. 49. Federal Reserve Board. Washington D.C.

Diebold, F. and Rudebusch, G. (1989) Long memory and persistence in aggregate output. Journal of Monetary Economics 24, 189-209.

Dolado, J. (1989) Optimal instrumental variable estimator of the AR parameter of an ARMA (1,1), mimeo, (forthcoming in Economic Theory).

Dolado, J. and Jenkinson, T. (1987) Cointegration: A survey of recent developments. Applied Economics Discussion Paper No. 39. University of Oxford

Engle, R. and Granger, G. (1987) Cointegration and error correction: Representation, estimation and testing. Econometrica 55, 251-276.

gre, R., Granger, C., Hylleberg, S. and Yoo, S. (1987) Seasonal integration and and YoO S. (1987) Forecasting and

Ecomometrics $35,143-159$.

Ecribano, A. (1987) Cointegrato

co-trends and error correction systems: An alternative approach. CORE Discussion Paper No. 8715. University of Louvain.
Fuller, W. (1976) Introduction to Statistical Time Series. New York: John Wiley and Sons.

Geweke, J. and Porter-Hudak, S. (1983) The estimation and application of long memory time series models. Journal of Time Series Analysis 4, 221-238.

Gilbert, C. (1986) Professor Hendry's econometric methodology. Oxford Bulletin of Economics and Statistics 48, 283-307.

Gonzalo, J. (1989) Comparison of five alternative methods of estimating long-run equilibrium relationships. Discussion Paper 89-55. University of California, San

Diego.
Granger, C. (1981) Some properties of time series data and their use in econometric model specification. Journal of Econometrics 28, 121-130.

economic variables. Oxford Bulletin of Economics and Statistics 48, 213-228.

(1988) Some recent developments in a concept of causality. Journal of Econometrics $39,199-211$.

Granger, C. and Newbold, P. (1974) Spurious regressions in econometrics. Journal of Econometrics 26, 1045-1066.

Granger, C. and Joyeux, R. (1980) An introduction to long memory time series model and fractional integration. Journal of Time Series Analysis, 1, 15-39.

Granger, C. and Weiss, A. (1983) Time series analysis of error correction models, in Karlin, S., Amemiya, T. and Goodman, L. (eds), Studies in Economic Time Series and Multivariate Statistics, New York: Academic Press.

Haldrup, N. and Hylleberg, S. (1989) Unit roots and deterministic trends, with yet another comment on the existence and interpretation of a unit root in U.S. GNP mimeo. University of Aarhus.

Hall, A. (1989). Testing for a unit root in the presence of moving average errors. Biometrika 76, 49-56.

, S. Engle and Granger two-step estimation procedure U.K. aggregate Oxford Bulletin of Economics and Statistics 48 ,

Hannan, E. (1970) Multiple Time Series. New York: Wiley.

Hansen, B. (1988) A model of heteroskedastic cointegration, mimeo. Yale University. Ansen, B. and Phillips, P. (1988) Estimation and inference in models of cointegration A simulation study. Cowles Foundation Discussion Paper No. 881. Yale University.

Harvey, A. (1981) Time Series Models. Oxford: Phillip Allan.

Hendry, D. (1986) Econometric modelling with cointegrated variables: An overview. Oxford Bulletin of Economics and Statistics 48, 201-212.

Hendry, D. and Mizon, G. (1978) Serial correlation as a convenient simplification not a nuisance: A comment on a study of the demand for money by the Bank of England. Economic Journal 88, 349-363.

Hendry, D. and Richard, J. F. (1983) The econometric analysis of economic time series. International Statistical Review 51, 111-163.

Hylleberg, S. and Mizon, G. (1989) A note on the distribution of the least square estimation of a random walk with drift. Economics Letters 29, 225-230.

Jenkinson, T. (1986) Testing neo-classical theories of labour demand: An application of cointegration techniques. Oxford Bulletin of Economics Statistics 48, 241-251.

Johansen, S. (1988) Statistical analysis of cointegration vectors. Journal of Economic Dynamics and Control 12, 231-254.

Johansen, S. and Juselius, K. (1988) Hypothesis testing for cointegration vectors with an application to the demand for money in Denmark and Finland. Working Paper No. 88-05. University of Copenhagen.

Molinas, C. (1986) A note on spurious regressions with integrated moving average errors. Oxford Bulletin of Economics and Statistics 48, 279-282. 
Nelson, C. and Kang, H. (1981) Spurious periodicity in inappropriately detrended time series. Econometrica $49,741-751$.

Nelson, C. and Plosser, C. (1982) Trends and random walks in macroeconomic time series. Journal of Monetary Economics 10, 139-162.

Newey, W. and West, K. (1987) A simple, positive semi-definite heteroskedasticity and

Nickell, S. (1985) Error correction, partial adjustment and all that: An expository note. Oxford Bulletin of Economics and Statistics 47, 119-129.

Osborn, D. (1988) Seasonality and habit persistence in a life cycle model of consumption. Journal of Applied Econometrics 3, 255-266.

Osborn, D. Chui, A Smith J and Birchenhall. C. (1988) Seasonality and the order of integration for consumption. Oxford Bulletin of Economics and Statistics 50 , $361-377$.

Ouliaris, S, Park, J, and Phillips, P. (1988) Testing for a unit root in the presence of a maintained trend (forthcoming in B. Raj (ed) Advances in Econometrics and Modelling. Needham: Kluwer Academic Press)

Pagan, A. and Wickens, M. (1989) A survey of some recent econometric methods. Economic Journal, 99, 962-1025.

Park, J. and Phillips, P. (1988) Statistical inference in regression with integrated processes: Part 1. Econometric Theory 4, 468-497.

Perron, P. (1987) The great crash, the oil price shock and the unit root hypothesis. Cahier 8749. University of Montreal (forthcoming in Econometrica).

Phillips, P. (1986) Understanding spurious regression in econometrics. Journal of Econometrics 33, 311-340.

(1987) Time series regression with a unit root. Econometrica 55, 277-301

(1988) Optimal inference in cointegrated systems. Cowles Foundation Discussion Paper No. 866. Yale University.

Phillips, P. and Ouliaris, S. (1988) Testing for cointegration using principal components methods. Journal of Economic Dynamics and Control 12, 205-230.

Phillips, P. and Perron, P. (1988) Testing for a unit root in time series. Biometrika 75 , 335-346.

Pierce, D. (1976) Seasonality adjustment when both deterministic and stochastic seasonality are present, in A. Zellner (ed) Seasonal Analysis of Economic Tim id S. Wnd Dickey, D. (1984) Testing for unit ro

Said, S. and Dickey, D. (1984) Testing for unit roots in autoregressive-moving average

(1985) Hothesis Statistical Association 80, 369-374.

Saikkonen, P. (1989) Asymptotically efficient estimation of cointegration regressions. Research Report No. 72, University of Helsinki.

Sargan, J. (1964) Wages and prices in the UK: A study in econometric methodology, in Hart, P. Mills, G. and Whittaker, J. (eds.) Econometric Analysis for National Planning, London: Butterworths.

Schwert, G. (1987) Effects of model specification on tests for unit roots in macroeconomic data. Journal of Monetary Economics 20, 73-103.

(1989) Tests for unit roots. A Montecarlo investigation. Journal of Business and Economic Statistics 7, 147-159.

Sims, C., Stock, J. and Watson, M. (1986) Inference in linear time-series models with

some unit roots, mimeo (forthcoming in Econometrica).
Sosvilla-Rivero, S. (1989) Cointegration and unit roots: A survey. Discussion Paper 89-5, University of Birmingham.

Stock, J. (1987) Asymptotic properties of least squares estimators of cointegrating vectors. Econometrica 55, 381-386.
Stock, J. and Watson, M. (1987) Variable trends in economic time series. Journal of Economic Perspectives 2, 147-174.

(1988) Testing for common trends. Journal of the American Statistical Association. 3, $1094-1107$.

Tiao, G. and Grupe M (1980) Hidden periodic autoregressive-moving average models in time-series data. Biometrika 67, 365-373.

West, K. (1988) Asymptotic normality when regressors have a unit root. Econometrica 56 , 1397-1417.

Wold, $H$ (1938) A study in the Analysis of Stationary Time-Series. Stockholm: Almguist and Wiknell. 